\title{
Entrelacs
}

Cinéma et audiovisuel

\section{L'alchimie poétique d'Alejandro Jodorowsky}

"Poesía, alumbrarás mi camino como una mariposa que arde".

Joy Courret

\section{OpenEdition}

\section{Journals}

Édition électronique

URL : http://journals.openedition.org/entrelacs/5627

DOI : $10.4000 /$ entrelacs.5627

ISSN : 2261-5482

Éditeur

Éditions Téraèdre

Référence électronique

Joy Courret, «L'alchimie poétique d'Alejandro Jodorowsky », Entrelacs [En ligne], 16 | 2019, mis en ligne le 06 novembre 2019, consulté le 05 décembre 2019. URL : http://journals.openedition.org/entrelacs/ 5627 ; DOI : 10.4000/entrelacs.5627

Ce document a été généré automatiquement le 5 décembre 2019

Tous droits réservés 


\title{
L'alchimie poétique d'Alejandro Jodorowsky
}

"Poesía, alumbrarás mi camino como una mariposa que arde".

\author{
Joy Courret
}

\section{Introduction}

1 La poésie est une discipline au cœur des arts chez Alejandro Jodorowsky, or on constate qu'elle reste très nettement en marge dans la biographie de l'auteur, où les films et les bandes dessinées sont souvent cités. Sa poésie évolue entre tradition et nouveauté, entre héritages et ruptures, et trouve sa place entre plusieurs sphères. Jodorowsky se démarque par ses inspirations surréalistes mais aussi par son attachement aux pensées orientales. En effet, l'artiste chilien présente des similitudes avec des auteurs comme René Daumal, Arthur Rimbaud, Fernando Pessoa ou encore certains penseurs chinois comme Tchouang Tseu ou Lao Tseu que Jodorowsky ne manque pas de citer dans ses œuvres. Nous verrons brièvement comment ses liens se tissent dans sa poésie.

2 La pluridisciplinarité de l'œuvre d'Alejandro Jodorowsky n'est plus à démontrer. Le parcours du poète chilien l'a amené à connaître divers mouvements littéraires et à rencontrer différentes personnalités. Alors qu'il arrive en 1953 à Paris, il trouve sa place dans le cercle des surréalistes, fait la connaissance d'André Breton et d'autres artistes puis décide de s'émanciper de ce groupe pour suivre sa propre voie. Il la trouvera dans les années 1960 avec la création du mouvement éphémère Panique aux côtés de Fernando Arrabal et Roland Topor. Ses productions cinématographiques majoritairement citées et étudiées attestent de ces années de réflexions artistiques engendrées par Panique. Ses collaborations avec des dessinateurs (Moebius pour n'en citer qu'un dans des projets tel L'Incal ${ }^{1}$ ) viennent compléter un tableau artistique dense et riche que l'auteur compose depuis les années 1950. Alejandro Jodorowsky transpose la poésie dans ses films et tire de son parcours des éléments qu'il reprend dans ses œuvres. 
3 Nous nous centrerons ici plus spécifiquement sur la poésie de l'auteur, et pour cela nous verrons comment se construit son discours poétique et les liens qu'il entretient avec l'alchimie, discipline ésotérique très présente dans de nombreuses productions de Jodorowsky. Son dernier film en date Poesía sin fin (2016) est un exemple qui illustre parfaitement la manière dont il se plait à combiner tous les arts et à donner naissance à des œuvres uniques, en mariant plusieurs formes d'expression. Dans ce film, les décors de théâtre abondent, pour rester fidèle à cet art qu'il pratique depuis la création du théâtre El Bululú ${ }^{2}$ au Chili ou encore au Mexique dans les années 1970. La poésie est bien entendu très présente dans cette dernière production cinématographique, ne serait-ce que par le titre très évocateur qui est aussi celui donné à l'anthologie poétique que Jodorowsky publie en $2013^{3}$ et qui regroupe tous ses recueils antérieurs, notamment No basta decir ${ }^{4}$, La Escalera de los ángeles ${ }^{5}$, Pasos en el vacío ${ }^{6}$ ou encore Yo el tarot $^{7}$.

4 La poésie de Jodorowsky se nourrit de tout son parcours et de ses rencontres littéraires, elle dépasse d'ailleurs le champ du simple poème. Elle est partout dans son œuvre et enrichit ses créations.Il est très jeune lorsqu'il découvre la poésie et se passionne pour la lecture. Son affection pour les poètes (Federico García Lorca, Pablo Neruda, Nicanor Parra, Charles Baudelaire, Vicente Huidobro, etc.) permet-elle d'affirmer que Jodorowsky appartient à une tradition poétique alchimique, à laquelle aujourd'hui on rattache volontiers Arthur Rimbaud, Gérard de Nerval, Fernando Pessoa ou encore Ibn Arabi ${ }^{8}$ ?

Dans un premier temps, nous verrons comment Alejandro Jodorowsky pense la poésie et pourquoi elle est essentielle pour lui. Nous interrogerons en deuxième partie ce concept d'alchimie et la façon dont cette science surgit dans ses recueils. Enfin, nous constaterons comment la poésie et l'alchimie se mêlent dans ses vers.

6 À se concentrer sur un aspect de l'œuvre d'Alejandro Jodorowsky, on s'aperçoit rapidement que chaque domaine artistique en appelle un autre et que chaque création fait résonner une autre création. Les arts ainsi entremêlés rendent la production de Jodorowsky bien particulière et l'étude d'un domaine revient finalement à s'intéresser à l'ensemble. Nous verrons au travers de ce texte que la poésie, bien qu'en retrait, se trouve finalement au centre de sa création et de son œuvre.

\section{Poésie et pensée poétique}

7 Alejandro Jodorowsky, au-delà de ses pratiques artistiques diverses, se définit avant tout comme poète ${ }^{9}$. C'est très jeune qu'il découvre la poésie et se lance dans une réflexion ${ }^{10}$ sur le genre poétique et sur l'art. La poésie est rapidement devenue un axe majeur et central qui le guidera dans ses créations futures.

\section{La poésie comme acte}

8 Le Chili aura joué un rôle capital dans la découverte de l'art poétique. Jodorowsky dit lui-même dans Psicomagia ${ }^{11}$ que le Chili est un pays hautement poétique, en attestent des figures comme Pablo Neruda, Raúl Zurita ou Gabriela Mistral.

En 1939, Alejandro Jodorowsky s'installe à Santiago avec sa famille et quitte alors Tocopilla. Durant cette période, il rencontre des artistes qui vont s'avérer devenir eux- 
aussi des auteurs reconnus en Amérique latine. Le poète Enrique Lihn, qui a exercé son art au Chili ainsi qu'à Cuba, est rapidement devenu ami avec le jeune Jodorowsky, et tous deux se sont livrés à des jeux poétiques ${ }^{12}$ que l'on voit mis en scène dans Poesía sin fin. Ces actes mêlant poésie et performances sont des expérimentations que Lihn et Jodorowsky baptisent "actes poétiques ». Dans Poesía sin fin, Enrique Lihn, interprété par l'acteur Leandro Taub, occupe un rôle important dans cette période d'adolescence. Dans Psicomagia, autobiographie sur sa vie dont on perçoit les échos dans le film Poesía sin fin, on lit cette complicité en ces termes:

J'avais la chance d'avoir le même âge que le célèbre poète Enrique Lihn, aujourd'hui décédé. Un jour, avec lui et quelques amis, nous avons trouvé dans un livre sur le futurisme italien une phrase éclairante de Marinetti : «La poésie est un acte ». À partir de cet instant, nous avons décidé de prêter plus attention à l'acte poétique qu'à l'écriture même. Pendant trois ou quatre ans, nous nous sommes dédiés à la réalisation d'actes poétiques. Nous y pensions toute la journée ${ }^{13}$.

Alejandro Jodorowsky et Enrique Lihn se dédiaient à des actes poétiques, qui consistaient à rendre leurs gestes et leurs journées plus sensibles à l'environnement, diffusant ainsi l'art au-delà des simples vers. Jodorowsky prend en exemple cet acte marquant que l'on retrouve à l'écran en 2016 dans Poesía sin fin :

Par exemple, Lihn et moi avions décidé de marcher en ligne droite sans jamais dévier notre trajectoire. Nous marchions sur une avenue face à un arbre. Au lieu de le contourner, nous avons grimpé à l'arbre pour continuer notre conversation ${ }^{14}$.

11 Jodorowsky a hérité son besoin de libération poétique et artistique d'un autre poète chilien, disparu en 2018, Nicanor Parra. Ce professeur de mathématiques et poète est devenu une figure primordiale dans le panorama littéraire chilien. La figure de « l'antipoète $\aleph^{15}$ qu'a créée Nicanor Parra a séduit le jeune poète Alejandro. Pour Parra, qui rompt avec la figure nationale de Pablo Neruda, la poésie était partout, même dans les choses les plus simples, voire les plus insignifiantes. Au début des années 1950, à Santiago, Parra, Lihn et Jodorowsky ont joint leur créativité pour élaborer des œuvres murales, composées de collages de journaux ou de poèmes, intitulées les " Quebrantahuesos». Ce terme unit le vocable "quebrar» signifiant "briser», et " huesos ", traduction du substantif «os ». Ces affiches murales éphémères étaient destinées à attirer le regard des passants qui marchaient dans les rues de la ville. Entre l'information et le poétique, Lihn, Jodorowsky et Parra entendaient bien détruire les barrières et se jouer des journaux de l'époque en les imitant, à la manière du pastiche.

Alejandro Jodorowsky a alors véritablement pensé la poésie comme une pratique artistique mais aussi comme un acte, un art qui doit être vécu et expérimenté, qui le guide dans ses productions. Comme il le dit dans son anthologie poétique : «Je ne crois pas en la révolution politique, je crois en la révolution poétique ${ }^{16}$. $"$

Dans ses productions cinématographiques, la poésie naît avec les mouvements, les couleurs et les références. Dans La Montagne sacrée, Jodorowsky laisse s'échapper des oiseaux du torse des morts pour, semble-t-il, symboliser l'âme. L'ambiance du film tout entière laisse suggérer une poésie omniprésente. En se transformant en un poète guide à la fin du film et à la manière d'un performeur, Jodorowsky le réalisateur porte à l'écran Jodorowsky le poète. L'union entre l'art poétique et l'art cinématographique apparaît très simplement dans le nom du film Poesía sin fin, poésie sans fin, sans bornes donc. Poesía sin fin embrasse totalement ce désir d'unir chaque œuvre, chaque production et chaque art autour de la poésie. 


\section{La poésie chinoise, une autre perspective et une inspiration nouvelle pour la poésie de Jodorowsky}

Si les auteurs et poètes chiliens ont été une source d'inspiration puissante et fertile, on peut également retrouver des échos de la spiritualité orientale dans l'œuvre de Jodorowsky. Très curieux de spiritualité, Jodorowsky va s'éveiller à la philosophie et à la pensée orientales au contact d'un moine Zen japonais, Ejo Takata. Ce Maître, qu'il rencontre lors de son voyage au Mexique dans les années 1970, va lui ouvrir la voie de ces pensées issues de la spiritualité japonaise, et plus largement asiatique. En découvrant le bouddhisme, Jodorowsky s'intéressera par la suite à des piliers de la philosophie chinoise, tels le confucianisme ou le taoïsme. Alejandro Jodorowky fait de son œuvre un lieu d'expression et de représentation de ces principes, et ce plus particulièrement dans l'œuvre poétique. Ce poème témoigne de l'importance que le poète accorde à cette pensée épurée :

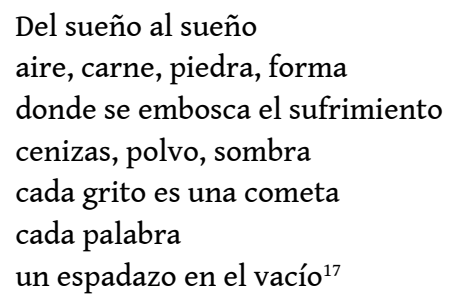

La pensée et la philosophie chinoises, notamment le taoïsme et la poésie de la dynastie Tang ${ }^{18}$, sont, au même titre que le surréaliste français René Daumal ${ }^{19}$ ou la philosophie Hermétique $^{20}$, des influences poétiques profondes pour le poète.

Dans Psicomagia, Alejandro Jodorowsky met en relation la Chine et le Chili, les dévoilant comme étant toutes deux des nations poétiques, réaffirmant ainsi son attachement pour son pays natal et pour l'Orient qu'il admire:

Dans combien de pays existe-t-il une atmosphère vraiment poétique? Sans nul doute, la Chine Antique était une terre de poésie. Mais je pense que, dans les années 50 au Chili, on vivait poétiquement comme dans aucun autre pays au monde ${ }^{21}$.

La voix poétique d'Alejandro Jodorowsky converse librement avec les penseurs ou leur rend hommage de plusieurs façons, par le biais d'un vers, d'un poème ou d'un mot qui laissent résonner les principes taoïstes par toute la symbolique qu'ils dégagent. Un recueil d'Alejandro Jodorowsky est entièrement dédié à la poésie chinoise, plus précisément à la poésie de la dynastie des Tang. Cet ouvrage, intitulé Imagen del alma ${ }^{22}$, se présente comme un entretien en vers libre. Jodorowsky, comme il l'annonce dans la préface, entend redonner une voix à ces poètes d'un autre temps. Les poètes Tang étaient des poètes taoïstes, soucieux de représenter les préceptes de leur philosophie dans leurs vers. Chaque poème tiré de Imagen del alma porte le nom d'un sage ou d'un poète. Le Je lyrique dans ces poèmes exaltera un sentiment, comme la paix ou le bonheur. Voici le poème «VIII » que Jodorowsky destine à King Kien, qui semble être un poète imaginaire :

VIII King Kien- Fuerza dócil

Se desplaza como el espíritu en un mundo vacío de obstáculos. Se

propaga como un soplo desplegando el arco iris.

Construye castillos en el aire y es capaz de vivir en ellos.

Pisa las nubes y se aleja con el viento. Se nutre de vacío, ese es

el secreto de su fuerza ${ }^{23}$. 
Dans ce poème, le vide envahit tout, comme nous le montre le lexique : «vacío » (vide) au vers un, «soplo» (souffle) au vers deux, « aire» (air) «nubes» (nuages), ou encore « viento " (vent) aux vers trois et quatre. Dans le vers quatre, "Se nutre de vacío " (que l'on pourrait traduire par «Il se nourrit de vide ») confirme très simplement l'importance du vide dans le poème, élément que l'on peut rattacher au taoïsme et à ses principes. Le terme de taoïsme s'est appliqué d'abord aux écrits de certains mystiques de la Chine antique. Le Dao (ou Tao comme nous pouvons parfois le trouver) est le Principe régulateur de l'Univers, il est un principe de perfection. Le Dao est la Voie, sousentendu la bonne voie à suivre pour atteindre l'absolu et la perfection. Les penseurs chinois adeptes de cette pensée estimaient que l'harmonie universelle dépendait des manifestations du Dao. La Voie est la volonté supérieure et divine qui régit le temps et l'univers. La poésie d'Alejandro Jodorowsky est une poésie de la contemplation, elle semble tendre à représenter ces principes taoïstes, telle la plénitude du Vide, que l'on constate dans le poème précédent, l'inaction ou encore le détachement. Le " non agir ", la Voie et la plénitude du vide sont exposés dans le Tao Te King ${ }^{24}$, attribué à Lao Tseu et datant du IV $\mathrm{IV}^{\mathrm{e}}$ siècle avant J.-C.

Lorsque l'on étudie les poèmes d'Alejandro Jodorowsky, plusieurs choses interpellent, notamment la multiplicité de certains termes à la symbolique très forte. Le terme de miroir, "Espejo ", apparaît à de nombreuses reprises dans ses poèmes. Le miroir, considéré communément comme le reflet de l'inconscient, nous montre comment l'âme et l'imagination occupent une place prédominante dans la poésie de Jodorowsky :

\section{Exemple 1}

Soy la bestia que exige un territorio cuando cortas las raíces.

Sin embrago eres mi obra, te seguiré creando

porque eres tú la esperanza que arde en mí, el espejo ${ }^{25}$

Exemple 2

Cuando entrego la mirada que mira a la mirada

con un suspiro de reposo me transformo

en una imagen que rechaza los espejos ${ }^{26}$

Exemple 3

parece que soy una horrible ciudad de espejos

parece que soy un ejército de sombras

parece que me he convertido en as de copa ${ }^{27}$

Dans le premier extrait du poème "Voz del espejo ", le Je poétique est un créateur qui s'adresse à son œuvre qu'il interpelle grâce à " eres mi obra » au vers dix du poème. Le miroir est aussi un moyen de rejeter et de repousser son Je dans la poésie. C'est le cas dans le poème "Vivir » dont on peut lire les vers six, sept et huit dans le deuxième exemple. La voix poétique joue avec l'ambivalence regard-miroir. L'allitération présente dans « mirada que mira a la mirada » centre l'intérêt du lecteur sur la vue et sur l'importance du regard. Dans le deuxième exemple, on comprend que ce miroir est associé au refus dans le vers suivant: "en una imagen que rechaza los espejos ». C'est l'image même du Je poétique qui rejette le miroir ici, et, tel un miroir inversé, l'image ne se réverbère pas. L'image n'est pas le résultat d'un reflet, le Je du poète n'est pas reflété mais réfuté. Dans ces trois extraits de poèmes, le miroir dévoilé dans les vers est souvent différent et se rapporte à des symboles opposés. Le miroir, par l'image symétrique qu'il renvoie, produit le reflet mais aussi tout son contraire. Il est symbole de double, de mensonge, tout comme de vérité. Si les symboles taoïstes, tels le célèbre Yin et Yang $^{28}$ ou encore l'exaltation du vide, sont puissants dans la poésie de Jodorowsky, l'idée de renaissance et de renouveau, que l'on retrouve dans la 
philosophie orientale et l'alchimie, confère à ses poèmes une certaine teneur philosophique. L'idée de renaître spirituellement est une idée partagée par les différents courants spirituels dans la Chine antique ${ }^{29}$.

Nous notons que dans ces pratiques taoïstes, on peut déceler des idées alchimistes. L'alchimie apparaît dès l'Antiquité et devient une science occulte très pratiquée au Moyen-Âge. Elle surgit dans toutes les cultures ; ainsi, il existe des alchimies grecque, occidentale, chrétienne et arabe. Pour simplifier, nous parlerons de la notion de métamorphose qu'implique l'alchimie. Les doctrines taoïste et alchimique partagent cette notion de transformation, une transformation progressive et immuable de l'esprit que l'on découvre dans les vers d'Alejandro Jodorowsky. Par transformation, nous comprenons tout processus de métamorphose, physique ou mental. Le poème précédent, "Vivir», en est un parfait exemple. La philosophe française Françoise Bonardel, spécialiste de l'alchimie et de l'Hermétisme, confirme cette union entre spiritualité asiatique et alchimie. Dans l'article «L'alchimie, philosophie par le feu », on peut lire :

Finalement plus proche des voies asiatiques (taoïsme, bouddhisme tantrique) que du dualisme occidental opposant matière et esprit, la pratique alchimique revient à extraire la quintessence des quatre éléments (eau, air, terre, feu), soumis à une rotation incessante dans le vase de verre (matras), dont le "fils d'Hermès » redoutent plus que Tout l'éclatement ${ }^{30}$.

L'alchimie est une notion que nous devrons au préalable définir tant elle est obscure et parfois folklorique. Le « fils d'Hermès » symbolise la philosophie Hermétique qui tend à allier toutes les cultures et à révéler des savoirs universels. Françoise Bonardel décèle des similitudes intéressantes entre Hermétisme et spiritualité asiatique, deux pensées que l'on identifie au sein de la poésie de Jodorowsky. Dans la pensée taoïste, la vie résulte de la combinaison du Yin et du Yang, c'est à dire d'un équilibre ${ }^{31}$. On peut lire dans ce Yin et ce Yang orientaux, un équivalent de l'alchimie occidentale qui oppose la matière vile et l'or. Alejandro Jodorowsky, inspiré de ces deux voies orientale et occidentale, fait un pont entre ces deux cultures, pont qu'il rend visible dans son art poétique. Pour Françoise Bonardel, l'Hermétisme est une pensée philosophique unissant plusieurs religions, philosophies et cultures, qui bouscule la notion même de limite entre chacun de ces courants. L'alchimie est une branche de cette philosophie qui incite l'homme à se transformer, ou, dans une conception plus classique, changer les métaux vils en or $^{32}$. Le processus de transformation est donc le même dans l'alchimie et dans le taoïsme, s'il l'on en croit la spécialiste française.

Le fort symbolisme qui se détache de la poésie d'Alejandro Jodorowsky occupe donc un espace substantiel puisqu'il permet à des spiritualités parfois insaisissables de s'exprimer. Le mysticisme notamment ne parvient à devenir limpide que dans l'espace poétique. Les abîmes (abismos en espagnol) ou encore le miroir évoqué précédemment, reviennent constamment dans les vers, au même titre que certaines couleurs ou certaines lames du tarot. Les abîmes sont aujourd'hui associés à la mystique de Saint Jean de la $\mathrm{Croix}^{33}$. Son Je lyrique choisit de sonder les profondeurs de l'obscurité et symbolise les gouffres que suppose l'abîme à travers certaines métaphores et champs lexicaux, ou se réfère directement à l'abîme. Parmi les nombreuses occurrences du terme «abismo» dans les poèmes de Saint Jean de la Croix, on relève quelques occurrences poétiques :

5. 
Je sais bien que c'est un abîme sans fond

Et que personne ne peut y passer à gué

Quoique ce soit de nuit!

11.

Et pour qu'elle ait la vie

Je mourrai pour elle, Je la tirerai de l'abîme

Et je vous l'amènerai. ${ }^{34}$

L'abîme de Saint Jean de la Croix est bien ici un abîme profond, "sans fond", qui évoque tous les dangers. L'abîme de Jodorowsky, que l'on découvre ici par un court extrait, respecte cette idée de gouffre abyssal que l'on perçoit chez Saint Jean de la Croix :

En abismos infinitos vaga el pensamiento en busca de los límites que le darán dolor porque la conciencia necesita el sufrimiento

para no disolverse en la penumbra del amor. ${ }^{35}$

Le mysticisme regorge de références à l'abîme ou aux ténèbres. En ce sens, Jodorowsky à son tour s'emploie à renforcer cette image obscure et divine dans ces poèmes. Ce symbolisme lié à l'abîme est à la fois très marqué dans des sciences plus occultes qui évoluent aux côtés de la mystique, et au sein de la philosophie Hermétique, comme l'alchimie, que nous allons aborder plus longuement dans cette deuxième partie.

\section{Alchimie et poésie}

\section{La voie poétique}

Alejandro Jodorowsky se situe donc au carrefour de plusieurs pensées, de plusieurs philosophies et de plusieurs mondes. Entre l'Orient et l'Occident, sa poésie reflète des aspects divers de sa propre conception de la vie. La capacité de l'homme à se changer lui-même et à se bonifier est au centre du taoïsme. La Voie a cette vertu sacrée de pouvoir rendre l'âme pure, et pourquoi pas, immortelle ${ }^{36}$. C'est ici que l'alchimie prend une place considérable dans la poésie de Jodorowsky : l'alchimie poétique ou l'alchimie $\mathrm{du}$ verbe sont des termes que l'on pourrait associer autant à Jodorowsky qu'au mouvement surréaliste auquel il a pris part en arrivant en France. En effet, dans le Manifeste du surréalisme ${ }^{37}$ paru en 1924, on constate que Alchimie et Art sont mêlés dans la pensée surréaliste. André Breton préconise de laisser une grande place à l'imagination, à l'esprit et aux sciences occultes ${ }^{38}$. La publication d'Arcane $17^{39}$ en 1944 signe son attachement à l'ésotérisme. Dans son ouvrage, Breton tisse une longue métaphore poétique de l'amour en prenant pour guide l'Étoile, Arcane XVII du Tarot de Marseille. L'attrait du mystère justifie ce goût partagé pour l'occulte, par exemple pour le tarot et l'ésotérisme. Parallèlement, nombre de surréalistes se sont intéressés à la psychanalyse et aux possibilités créatives que cette nouvelle science offrait.

Jodorowsky célèbre l'alchimie et la représente dans ses vers comme nous l'avons vu précédemment. La science alchimique telle qu'on l'entend ici s'éloigne de la conception fantastique et imaginaire que l'on en a. Ce concept est considéré par Jodorowsky comme une science de perfectionnement de l'être en ce qu'elle permet à l'homme de se modifier, de changer son être profond par les arts et la pensée. On la dit ancêtre de la chimie, or ici nous considérons l'alchimie plutôt comme une branche de la philosophie 
Hermétique ${ }^{40}$. Philosophie et alchimie sont liées ne serait-ce que par la simple existence du mythe de la Pierre Philosophale, réputée pour changer les métaux vils en or.

Prenons des évocations de l'alchimie chez des auteurs consacrés tel Arthur Rimbaud qui parlait de l'alchimie du Verbe, dans le sens de la magie de la parole. C'est d'ailleurs dans un de ses poèmes de 1871 que l'on lit comment l'alchimie guide son inspiration poétique. Dans la seconde lettre appelée «La lettre du voyant », on peut lire :

Le Poète se fait voyant par un long, immense et raisonné dérèglement de tous les sens. Toutes les formes d'amour, de souffrance, de folie; il cherche lui-même, il épuise en lui tous les poisons, pour n'en garder que les quintessences ${ }^{41}$.

Arthur Rimbaud dans sa lettre au poète Paul Demeny du 15 mai 1871 développe l'idée d'une émergence d'une poésie nouvelle. La quintessence évoquée ici se réfère à la quintessence alchimique, l'élément qui assure la cohésion des quatre éléments fondamentaux. Le concept de transformation, de mutation évoqué précédemment, surgit lui aussi. Pour Rimbaud, la poésie doit tendre à une combinaison de plusieurs sens, synesthésie que l'on retrouve dans les vers de Jodorowsky. Dans « Magia de la vida » du recueil No Basta Decir, la vue se conjugue avec l'ouïe et le goût :

El paladar exquisito no tolera lamer piedras

y bajo el color del cruento día la mirada del pinto descubre gamas,

porque los sentidos continuamente dan a aquello que reciben ${ }^{42}$

Rimbaud fournit une réflexion sur le langage, sur la communication que suppose le genre poétique. Alejandro Jodorowsky, en suivant ce chemin-là, rapproche de nouveau la voie alchimique de la voix poétique.

L'alchimie est déjà au cœur de La Montagne sacrée. Le personnage principal, l'alchimiste, prend la responsabilité d'un groupe de huit hommes et femmes, désireux de laver leurs péchés et de devenir immortels, grâce à l'ascension de la montagne sacrée. Le seul motif de la montagne du film vient rappeler les montagnes mystiques (le Mont Meru, le Mont Sinaï ou encore le Mont Olympe), présentes dans toutes les civilisations. Par conséquent, ce motif est lié à l'ésotérisme et à la théorie des correspondances qu'il tisse ${ }^{43}$. Plus spécifiquement, dans la poésie de Jodorowsky, la montagne est un élément que l'on retrouve souvent: « Les montagnes entres les frontières se font labyrinthes ${ }^{44}$ », "Je suis parti au sommet d'une gigantesque montagne à la recherche de mon fils défunt ${ }^{45}$ ", ou encore "J'explore les flancs d'une mystérieuse montagne sans me préoccuper de la légende qui raconte qu'elle est habitée par de féroces guerriers en or ${ }^{46}$ ». La montagne devient un symbole inhérent à la poésie de Jodorowsky, de plus très ancré dans l'univers ésotérique auquel l'auteur se rapporte.

\section{Glissement vers la poésie philosophique}

Alejandro Jodorowsky, une fois arrivé à Paris en 1953, se rend au cours du philosophe français Gaston Bachelard à la Sorbonne. Cet enseignement va lui permettre de concevoir la poésie comme un art salvateur. La rêverie et la poésie sont pour Bachelard fortement liées, l'une est le reflet de l'autre et elles se complètent. Son travail sur l'alchimie semble avoir séduit Jodorowsky qui lui donne un rayonnent tout particulier dans sa poésie. Des œuvres telles La Poétique de la rêverie ${ }^{47}$ ou La Psychanalyse du feu ${ }^{48}$ montrent combien Bachelard est attaché à cette science alchimique, au sein même de l'art poétique. 

lors [...] la langue de l'alchimie est une langue de la rêverie, la langue maternelle de la rêverie cosmique ${ }^{49}$. » Poétique, rêverie, langage et alchimie évoluent ensemble et côte à côte dans la pensée de Bachelard, tout comme dans celle de Jodorowsky. La poésie qui découlera de cet enseignement, des années plus tard, sera le reflet de cette vision de l'art et de l'alchimie. Cette tradition alchimique est entretenue par Jodorowsky, dans ses vers et dans sa pensée. Prenons ce poème du recueil De aquello que no se puede hablar, nommé «Alquimia » où la voix poétique nous expose le processus de transformation alchimique :

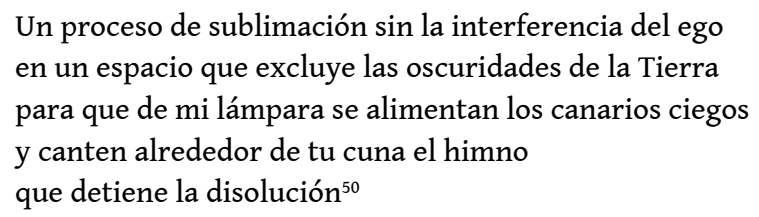

Ce poème offre une définition du fonctionnement de la science alchimique, en montrant bien l'importance de termes tels que : «sublimación » (sublimation) au vers un, «lámpara » (lampe) au vers trois, qui fait référence à la Gnose $\mathrm{e}^{51}$ et "disolución " (dissolution) au vers cinq. D'autres symboles que l'on associe à l'univers alchimique trouvent refuge dans les vers du poète: l'œuf, «huevo » en espagnol, tiré du vers " ¿Demolerás catedrales para exhumar el huevo donde yace un Mesías? $»^{52}$ ou encore "No existe nada más apasionante que el estudio del huevo ${ }^{53}$ ", fait référence à la naissance voire à la renaissance. Cette matrice qui permet à chacun de naître et renaître est fortement représentée dans la poésie de Jodorowsky.

Cette appartenance à des sphères plus ésotériques et des sciences occultes rapprochent alors l'artiste chilien d'un poète comme Baudelaire qui était lui aussi connu pour louer l'occulte dans ses vers. Rappelons ce quatrain des Fleurs $d u \mathrm{mal}^{54}$, où Satan et l'alchimiste se côtoient :

Sur l'oreiller du mal c'est Satan Trismégiste

Qui berce longuement notre esprit enchanté,

Et le riche métal de notre volonté

Est tout vaporisé par ce savant chimiste ${ }^{55}$

"Le riche métal de notre volonté » donne à penser aux métaux vils que l'alchimiste s'emploie à changer en or. L'allusion au chimiste de son côté est très parlante concernant cette science ancienne. Le poète portugais Fernando Pessoa entretenait lui aussi des liens avec l'ésotérisme. Cet homme de lettres bien mystérieux en plusieurs points a en effet exploré des dimensions occultes dans ses œuvres. Dans Le Livre de l'intranquillités, le Je poétique exalte des sciences « différentes ", perceptibles mais non visibles, et à son tour Alejandro Jodorowsky s'emploie à les exposer dans sa poésie. Nous prendrons en illustration ce quatrain de Pessoa :

Aveugle, la Science laboure la glèbe vaine.

Démente, la Foi vit le rêve de son culte.

Un Dieu nouveau n'est rien qu'un mot.

Ne cherche ni ne crois : tout est occulte ${ }^{57}$

La voix poétique évoque une science aveugle et terne, en terminant sur une touche occulte supposée révéler l'existence d'une vérité tout autre à son lecteur.

À son époque, Pessoa était plutôt isolé mais prolifique dans son écriture, tellement productif que son seul Je ne suffisait plus. De nombreux hétéronymes, en relations entre eux parfois, produisaient en toute indépendance des poèmes, romans et articles. 
Ce n'est qu'à la mort du poète que ces noms de la vie littéraire portugaise lui ont été associés. Ses hétéronymes étaient pour Fernando Pessoa des masques qui lui permettaient de jouer un rôle et de se cacher. Pessoa utilise en poésie l'image du miroir pour donner de la substance à cette quête d'identité et pour sortir de ce Moi devenu prison. Dans une note écrite en 1915, il explique : « je me sens multiple, je suis comme une chambre avec d'innombrables miroirs fantastiques qui déforment, en de faux reflets, une seule vérité antérieure ${ }^{58}$.»

\section{Yo el tarot, conversation du tarot et de la poésie} qui est en effet un moyen sûr pour le poète de représenter l'alchimie. Le tarot de Marseille appartient lui aussi à cette sphère ésotérique symboliste. En effet, les couleurs, les gestes et les attributs des lames sont tous des symboles et cet art ne repose que sur ces images et leurs interprétations. Dans son recueil Yo el tarot ${ }^{59}$, Jodorowsky conçoit vingt-deux poèmes pour les vingt-deux arcanes majeurs du tarot. L'attirance de Jodorowsky pour le tarot lui vient de l'étude de ce jeu au Chili. Pour l'artiste, le tarot n'est pas un art divinatoire mais un langage et un art de rétrospection qu'il étudie à partir de $1981^{60}$. "Arcane» étymologiquement signifie «coffre», ou encore «mystérieux $»^{61}$. La simple traduction du terme parle d'elle-même et nous invite à réfléchir sur le tarot et les possibilités qu'il offre, qu'elles soient poétiques ou psychanalytiques. Chaque carte est un outil que ne demande en somme qu'à être utilisé.

41 Ainsi le premier poème dédié au Mat, Arcane 0 dans le tarot, permet à l'Arcane de $s^{\prime}$ exprimer et de parler de ses caractéristiques et de son pouvoir ${ }^{62}$. Pour Jodorowsky, ces arcanes ont tous un pouvoir, un don mis à la disposition de celui qui tire les cartes ou qui reçoit le tirage. Chaque tirage est une phrase. Le tarot selon la conception de Jodorowsky est un livre qui va permettre à chacun de se comprendre et de débloquer une situation.

Le poème « El Loco » ${ }^{63}$, «Le Mat » en français, débute ainsi :

Peregrino en el encanto abominable de las formas

mensajero de lo esencial, es decir de mí msimo

desdeñando los ensueños del pensar

hago de todos los caminos mi camino. ${ }^{64}$

À travers la personnification du Mat, Alejandro Jodorowsky nous donne à lire et à comprendre les arcanes et leurs facultés. La poésie d'Alejandro Jodorowsky est à percevoir comme une quête spirituelle. Le Mat dans ce poème est le guide qui va déambuler dans tout le jeu et parmi toutes les cartes, c'est d'ailleurs la symbolique qu'on lui accorde lors d'un tirage. On l'observe vite dans le lexique: "peregrino" (pèlerin) au vers un, «mensajero » (messager) au vers deux, rehaussé par la répétition de « caminos " (chemin), aux vers trois et quatre, qui sous-entend un cheminement. La quête et la révélation qu'elle suppose alors nous rappelle des poètes romantiques, tel l'allemand Novalis, qui avait lui aussi un goût prononcé pour l'alchimique et le feu 
purificateur. Jodorowsky a à son tour fait du symbolisme poétique une voix d'imagination créatrice ${ }^{65}$.

Viaje esencial ${ }^{66}$ est le dernier recueil d'Alejandro Jodorowsky, et les poèmes n'échappent pas à cette tendance à unir deux arts, deux œuvres ou deux pratiques, en l'occurrence le symbole pictural du taoïsme à la poésie. De plus, ce recueil paru en 2016 est illustré par Pascale Montandon Jodorowsky, sa compagne, elle aussi artiste, photographe et écrivain.

Viaje esencial se compose de quatre parties. La troisième, «A la sombra del Yi King », se lit selon le tirage du Yi -King chinois, qui est un système codifié de tirage divinatoire, proche du tarot dans le principe mais différent dans le tirage et dans les symboles. Il se compose de soixante-quatre symboles, appelés les hexagrammes. Aussi connu sous le nom de "Livre des transformations ", le Yi King est pour ce recueil de Jodorowsky une clef de lecture. Avec cette récente publication, Alejandro Jodorowsky unit une nouvelle fois poésie, alchimie et spiritualité orientale. De plus, Carl Gustav Jung, psychiatre suisse souvent repris par Jodorowsky notamment dans ses ouvrages sur le $\operatorname{tarot}^{67}$, semble avoir tiré sa théorie de la synchronicité de l'étude du Yi King ${ }^{68}$. Le poème qui suit correspond à l'hexagramme 15 du Yi King qui s'intitule « Humilité » :

15

Libertad interior

Preso en la ley común, sé libre en tus entrañas.

Prohibiciones y órdenes florecen en las superficies, dentro

de tu cráneo reside lo insondable.

La conciencia es una barca que navega en el mar de la locura.

Porque eres mortal eres libre.

Que en tu cuerpo encadenado dance el esqueleto !

Amamantado con morales pétreas ¿sabrás disolverte en lo

infinito ?69

Le Je poétique utilise l'hexagramme de l'Humilité sous le nom de "Libertad interior » pour en proposer son interprétation poétique. Le lexique employé dans ces vers amène cette idée d'intériorité dès le premier vers avec "Preso en la ley común", "dentro/de tu cráneo reside lo insonsable " au troisième vers, et "en tu cuerpo encadenado dance el esqueleto » au vers sept. L'impératif «sé » au vers un rappelle l'aspect divinatoire de cet ensemble de signes, tout en établissant un dialogue entre le Je poétique et un $\mathrm{Tu}$.

Tout au long des soixante-quatre poèmes, le Je lyrique propose au lecteur une adaptation de chaque hexagramme. Ainsi, on peut imaginer un tirage classique du Yi King où les symboles se lieraient aux poèmes, donnant ainsi une clef d'interprétation supplémentaire.

\section{Conclusion}

Alejandro Jodorowsky, par sa capacité à créer dans des domaines différents, se place dans un discours d'art total ${ }^{70}$. Ses relations avec les surréalistes, son appartenance au mouvement Panique ou encore son héritage chilien hautement artistique rendent son œuvre parfois inclassable. Les créations se complètent et s'organisent en une symphonie colorée et sensible. L'immersion rapide dans l'art poétique l'invite progressivement à s'intéresser aux sciences dites occultes et plus particulièrement à l'alchimie, qui deviendront des sources d'inspiration puissantes pour sa poésie et pour son œuvre entière. L'alchimie est une notion clef dans son discours poétique: les 
références, les échos à d'autres poèmes et le symbolisme permettent à la voix poétique de construire et de s'exprimer pleinement. La dualité est l'un des éléments qui cristallisent la manifestation de l'ésotérisme dans la poésie de Jodorowsky. Le Je lyrique vacille entre le bien et le mal, la sacré et le profane, l'invisible et le visible, la religion ou encore l'athéisme ${ }^{71}$.

Alejandro Jodorowsky s'inspire en grande partie de la philosophie et de la spiritualité chinoises qui font jouer une chose et son contraire : l'absence et la présence, le vide et le plein, l'action et l'inaction. C'est en usant de ces binarités-là que Jodorowsky parvient, dans ses poèmes, à nous donner à voir l'invisible et l'absence, et à rendre le silence palpable.

S'il est parfois difficile de situer artistiquement Jodorowsky, qui déambule entre le surréalisme, les avant-gardes et le mouvement Panique, il est certain cependant que son œuvre, son Grand Euvre, pour reprendre un terme alchimique qui semble ici adéquat, reste unique. La poésie qu'il expérimente très jeune reste le point de départ de son œuvre. Au Chili, elle a d'abord été pensée comme un "acte ", puis cet art n'a plus jamais quitté l'auteur, qui l'incorpore à chaque production depuis lors.

51 La poésie alchimique de Jodorowsky vise à unir tous les arts. C'est finalement cette union entre les cultures, les œuvres et les pratiques, qui définit la notion même d'ésotérisme dans l'œuvre de Jodorowsky ${ }^{72}$. L'unité parfaite qui découle de l'alchimie et par conséquent de l'ésotérisme est à atteindre, et Alejandro Jodorowsky s'emploie à toucher une certaine unité artistique en plaçant, en son cœur, la poésie.

\section{NOTES}

1. Alejandro Jodorowsky et Moebius, L'Incal, Paris, Les Humanoïdes associés, 1981-1988.

2. Diego Moldes, Alejandro Jodorowsky, Madrid, Catedra, 2012, p. 90. Cette troupe de théâtre prend vie au sein du Teatro de la Universidad de Chile. Ces expérimentations théâtrales et poétiques sont la base de sa pensée artistique aujourd'hui.

3. Alejandro Jodorowsky, Poesía sin fin, Barcelone, Debolsillo, 2013.

4. Alejandro Jodorowsky, No basta decir, Madrid, Visor, 2000 ; Dire ne suffit pas : no basta decir, trad. Martin Bakero, David Giannoni, Emmanuel Lequeux, Paris, Le veilleur, 2003 pour la traduction française.

5. Alejandro Jodorowsky, La Escalera de los ángeles, Madrid, Obelisco, 2000 ; Alejandro Jodorowsky, L'Échelle des anges. Un art de penser, trad. Marianne Costa, Denis Patouillard-Demoriane, Paris, Albin Michel, 2001, pour la traduction française.

6. Alejandro Jodorowsky, Pasos en el vacío, Madrid, Visor, 2009.

7. Alejandro Jodorowsky, Yo el tarot, Madrid, Siruela, 2004.

8. Omari Hammami, Patricia Mons, Le Grand Diwan - Ibn 'Arabî, Paris, Albin Michel, 2016.

9. Dans le film Poesía sin fin, le jeune Jodorowsky annonce à sa famille son souhait de devenir poète. En de nombreuses occasions Alejandro Jodorowsky se place en poète, notamment lors de conférences publiques.

10. Diego Moldes, Alejandro Jodorowsky, op. cit., p. 89.

11. Alejandro Jodorowsky, Psicomagia, Buenos Aires, Debolsillo, 2008, p. 30. 
12. Dans La Danza de la realidad, Alejandro Jodorowsky accorde une place majeure à ces jeux poétiques durant lesquels lui et Enrique Lihn se fixaient un objectif d'acte poétique à réaliser dans la journée. Cela pouvait se résumer à se rendre à une conférence et à jeter de la viande fraîche sur le public. Cette scène apparaît dans le film Poesía sin fin. Dans Psicomagia, il raconte comment avec Enrique Lihn, ils ont mis en scène un acte poétique qui consistait à organiser la fausse veillée mortuaire de la mère de Jodorowsky. En rentrant chez elle, elle a donc découvert un mannequin vêtu de ses vêtements allongé dans son lit (Alejandro Jodorowsky, La Danse de la réalité, Paris, Albin Michel, 2004, p. 37).

13. Alejandro Jodorowsky, Psicomagia, op. cit., p. 35 : « Tuve la suerte de tener la misma edad que el famoso poeta Enrique Lihn, hoy fallecido. Un día, con él y otros compañeros, encontramos en un libro sobre el futurismo italiano una frase iluminadora de Marinetti : "La poesía es un acto". A partir de ese momento, decidimos prestarle más atención al acto poético que a la escritura misma. Durante tres o cuatro años, nos dedicamos a realizar actos poéticos. Pensábamos en ellos durante todo el día. » (traduction personnelle).

14. Ibid., "Por ejemplo, Lihn y yo decidimos un día caminar en línea recta, sin desviarnos nunca. Caminábamos por una avenida y llegábamos frente a un árbol. En vez de rodearlo, nos subíamos al árbol para proseguir nuestra conversación » (traduction personnelle).

15. Nicanor Parra invente ce terme conjointement à la publication en 1954 de son recueil Poemas $y$ antipoemas à Santiago aux éditions Nascimiento, qui changea la manière de penser la poésie en Amérique latine. Le succès de ce recueil a fait de Parra un (anti-)poète de renom.

16. Alejandro Jodorowsky, Poesía sin fin, op. cit. Cette mention apparaît en quatrième de couverture: "Yo no creo en la revolución política, yo creo en la re-evolución poética. " (traduction personnelle).

17. Alejandro Jodorowsky, «Sin meta », Pasos en el vacío, dans Poesía sin fin, op. cit., p. 390, v. 1-8 : «Du songe au songe/air, chair, pierre, forme/ où s'embusque la souffrance/cendres, poussières, ombres/chaque cri est une comète/chaque mot/un coup dans le vide » (traduction personnelle). 18. La dynastie des Tang émerge en Chine au début du VIIe siècle. Voir Pascal Buresi, entrée « DYNASTIE TANG », Encyclopædia Universalis, http://www.universalis.fr/encyclopedie/dynastietang/ (dernière consultation le 25 mai 2019). Isabelle Robinet, dans son ouvrage Histoire du taoïsme (Paris, Éditions du Cerf, 1991), souligne que sous la dynastie des Tang, le taoïsme vit une grande période d'épanouissement. Cette dynastie a en effet favorisé l'expansion de cette pensée.

19. Le film La Montagne sacrée, sorti en 1973, est une adaptation du roman Le Mont analogue de René Daumal, publié en 1952 aux Éditions Gallimard.

20. La philosophe Françoise Bonardel, dans La Voie hermétique (Paris, Dervy Poche, 2011), montre comment l'art hermétique cherche et œuvre à nouer, à « rétablir le contact » comme elle le dit entre l'homme et la Nature, le céleste et le terrestre. La philosophie Hermétique nous vient d'Hermès, dieu messager, et regroupe l'ensemble des doctrines ésotériques (alchimie, le rosicrucisme, le paracelsime ou encore la kabbale).

21. Alejandro Jodorowsky, «Acto poético », Psicomagia, op. cit., p. 30 : «¿En cuántos países existe una atmósfera realmente poética? Sin duda, la antigua China era una tierra de poesía. Pero pienso que, en los años cincuenta, en Chile se vivía poéticamente como en ningún otro país del mundo. » (traduction personnelle).

22. Alejandro Jodorowsky, Imagen del alma, dans Poesía sin fin, op. cit., p. 9-21.

23. Alejandro Jodorowsky, «VIII, King Kien Fuerza dócil », Imagen del alma, dans Poesía sin fin, op. cit., p. 14 : «Il se déplace comme un esprit dans un monde vide d'obstacles. Il/se propage comme un souffle déployant un arc-en-ciel./Il construit des châteaux dans les airs et est capable d'y vivre./Il marche sur les nuages et s'éloigne avec le vent, c'est cela/le secret de sa force.» (traduction personnelle).

24. Le Tao Te King (ou Dao de jing) a été classé parmi les textes taoïstes par les érudits de la dynastie Han. Il a une très grande influence en Orient et maintenant en Occident. Il traite des 
principes taoïstes tels l'amélioration de l'esprit, le «non agir », la vertu ou encore la Voie. "J'ai entendu dire que l'on comprend par la connaissance, mais pas encore qu'on pouvait comprendre par la non-connaissance. Du vide de l'esprit faillit la lumière : là se fixe le salut de l'homme.» Voir Jean-François Billetier, Études sur Tchouang Tseu, Paris, Éditions Allia, 2004, p. 112.

25. Alejandro Jodorowsky, «Voz del espejo », No basta decir, dans Poesía sin fin, op. cit., p. 182, v. 9-11: «Je suis la bête qui exige un territoire quand tu coupes les racines/Cependant tu es mon œuvre, je continuerai à te créer/parce que c'est toi l'espérance qui brûle en moi, le miroir.» (traduction personnelle).

26. Alejandro Jodorowsky, «Vivir ", Pasos en el vacío, dans Poesía sin fin, op. cit., p. 395, v. 6-8 : «Quand je donne le regard qui regarde le regard/dans un soupir de repos je me transforme/en une image qui rejette les miroirs. » (traduction personnelle).

27. Alejandro Jodorowsky, "Así va el mundo », No basta decir, dans Poesía sin fin, op. cit., p. 173, v. 8-10: «Il semble que je sois une horrible ville de miroirs/Il semble que je sois une armée d'ombres/Il semble que je me sois transformé en as de coupe ». (traduction personnelle).

28. Isabelle Robinet, Histoire du taoïsme, des origines au XIVe siècle, Paris, Éditions du Cerf, 1991, p. 14.

29. La philosophie taoïste se construit sur l'idée que chaque adepte tend à atteindre en suivant la voie du Tao. Voir Isabelle Robinet, Histoire du taoïsme, des origines au XIVe siècle, op. cit., p. 11.

30. Françoise Bonardel, "L'alchimie, la philosophie par le feu », dans 20 clés pour comprendre l'ésotérisme, Paris, Albin Michel, 2013, p. 60.

31. Isabelle Robinet, Histoire du taoïsme, des origines au XIVe siècle, op. cit., p. 15-16.

32. "L'alchimie aurait été une sorte d'art plus ou moins magique, consistant à combiner avec ingéniosité tours de passe-passe, cornues et invocations au Diable, dans le but d'obtenir de l'or, ou de passer pour en obtenir aux yeux des badauds émerveillés... Si l'alchimie n'avait été que cela durant toute la longue période où elle a été cultivée, elle ne mériterait certes pas d'avoir été étudiée par tant d'historiens et de savants modernes, à commencer par le grand chimiste Berthelot. Mais, lorsque l'on sait différencier les véritables alchimistes des escrocs et des charlatans qui prétendaient être des adeptes de l'art sacré, on s'aperçoit que l'alchimie, loin de se réduire à la simple fabrication de l'or, était en réalité quelque chose de beaucoup plus noble, et aussi de beaucoup plus complexe. » Serge Hutin, L'alchimie, Paris, PUF, « Que sais-je », 2005, p. 5.

33. Saint Jean de la Croix (1542-1591) était un prêtre carme, réformateur de son ordre et l'un des poètes mystiques espagnoles les plus notables.

34. Saint Jean de La Croix, « Malgré la nuit ", disponible en ligne : http://livres-mystiques.com/ partieTEXTES/JdelaCroix/poesies_st_jean_de_la_croix.pdf. (Consulté le 25 mai 2019).

35. Alejandro Jodorowsky, «En abismos infinitos vaga el pensamiento », De aquello que no se puede hablar, dans Poesía sin fin, op. cit., p. 72, v. 1-4: «Dans des abîmes infinies la pensée erre/à la recherche des limites qui lui donneront la douleur/parce que la conscience a besoin de la souffrance/pour ne pas se dissoudre dans la pénombre de l'amour » (traduction personnelle).

36. Isabelle Robinet, Histoire du taoïsme, des origines au XIVe siècle, op. cit., p. 55.

37. André Breton, La Manifeste du surréalisme, Paris, Éditions du Sagittaire, 1924.

38. Le désir de Breton de renouer avec le psychique de l'Humain est l'un des traits du surréalisme: «Le surréalisme vient ainsi désigner d'abord pour Breton cette pratique d'un certain automatisme psychique qui correspond assez bien à l'état de rêve : il s'agit alors de ne pas brouiller les messages élémentaires de l'inconscient en parasitant les associations ainsi produites par une quelconque élaboration stylistique ou par un contrôle conscient des productions libres de l'esprit». Philippe Sabot, "Primitivisme et surréalisme: une "synthèse" impossible?", Methodos [En ligne], 3 | 2003, mis en ligne le 05 avril 2004, consulté le 01 mai 2019. URL : http:// journals.openedition.org/methodos/109; DOI : 10.4000/methodos.109.

39. André Breton, Arcane 17, New York, Brentano's, 1944. 
40. Le Corpus Hermetica attribué à la figure mythique d'Hermès Trismégiste regroupe un ensemble de textes portant sur les doctrines considérées aujourd'hui comme hermétiques. Ces traités portent sur la médecine, l'astrologie, l'inspiration magique ou encore l'alchimie. Voir Françoise Bonardel, La Voie Hermétique, Paris, Dervy Poche, 2011, p. 9.

41. Arthur Rimbaud, Poésies complètes, Paris, Éditions de Poche, 2018, p. 141.

42. Alejandro Jodorowsky, « La magia de la vida », No basta decir, dans Poesía sin fin, op. cit., p. 162, v. 7-9: «Le palais exquis ne tolère de lécher la pierre/ et sous la couleur du jour sanglant le regard du peintre découvre des gammes,/parce que les sens continuellement donnent à ceux qui reçoivent. » (traduction personnelle).

43. Mircea Eliade, Le Sacré et le profane, Paris, Gallimard, «Idées », 1984, p. 39.

44. Alejandro Jodorowsky, Poesía sin fin, op. cit., p. 240, v. 16-17: «Montaña entre fronteras que se hacen laberintos ». (traduction personnelle)

45. Alejandro Jodorowsky, Poesía sin fin, op. cit., v. $1:$ « he ido hasta una altísima montaña en busca de mi hijo muerto ». (traduction personnelle).

46. Alejandro Jodorowsky, «Febrero », dans Poesía sin fin, op. cit., p. 153, v. 1 : « Exploro las faldas de una misteriosa montaña sin preocuparme de la leyenda que cuenta que está habitada por feroces guerreros de oro. " (traduction personnelle).

47. Gaston Bachelard, La Poétique de la rêverie, Paris, Presses Universitaires de France, 1960.

48. Gaston Bachelard, La Psychanalyse du feu Paris, Gallimard, 1938.

49. Gaston Bachelard, La Poétique de la rêverie, op. cit., p. 77.

50. Alejandro Jodorowsky, "Alquimia », De aquello que no se puede hablar, dans Poesía sin fin, op. cit., p. 35 : «Un processus de sublimation sans l'interférence de l'ego/dans un espace qui exclut les obscurités de la Terre/pour que de ma lampe s'alimentent les canaris aveugles/et qu'ils chantent autour de ton berceau l'hymne/qui possède (détient) la dissolution » (traduction personnelle).

51. La gnose est la connaissance que cherche à atteindre les adeptes par la pratique des sciences occultes. Cette connaissance est bien entendu cachée à la majorité et ne peut être révélée. La lampe, par la symbolique qu'elle renferme, a souvent été associée à une image renvoyant à cette recherche de la Gnose, de la connaissance supérieure (nous pensons par exemple à l'allégorie de la caverne). Elle fait également référence à la lanterne que tient l'Ermite, Arcane VIII du tarot. Cette lumière est celle de la connaissance et lui confère le rôle du guide savant.

52. Alejandro Jodorowsky, « La Fuerza », Yo el tarot, dans Poesía sin fin, op. cit., p. 247, v. 51-52.

53. Alejandro Jodorowsky, "Poesofía », De aquello que no se puede hablar, dans Poesía sin fin, op. cit., p. 30 , v. 51.

54. Charles Baudelaire, Les Fleurs du mal, Paris, Poulet-Malassis \& De Broise, 1857.

55. Charles Baudelaire, «Au lecteur », Les Fleurs du mal, Paris, Éditions de La Table Ronde, 1997, p. 5 .

56. Fernando Pessoa (attribué à Bernado Soares), o Livro do desassossego por Bernardo Soares, Lisbonne, Atica, 1982.

57. Fernando Pessoa, « Natal », Poesias, Lisbonne, Atica, 1967, p. 218, traduction issue de Cuvres de Fernando Pessoa (II), traduction de Michel Chandeigne et Patrick Quillier, Paris, Bourgeois, 1988, p. 26.

58. Ana Maria Binet, L'Ésotérisme dans l'œuvre de Fernando Pessoa, Livre I, thèse de doctorat dirigée par Marie-Hélène Piwnik, Université Bordeaux 3, soutenue en 1996, p. 167.

59. Alejandro Jodorowsky, Yo el tarot, Barcelone, Debolsillo, 2006.

60. Diego Moldes, Alejandro Jodorowsky, op. cit., p. 99.

61. Gérard Chandès, entrée "Arcane" dans Dictionnaire historique de la magie et des sciences occultes, Paris, Le livre de poche, 2006, p. 68.

62. Le Mat, selon Alejandro Jodorowky dans La Vía Del Tarot (Madrid, Siruela, 2011), représente « l'énergie sans limites, la liberté et l'impulsivité créatrice. Il est également un voyageur, tous les 
chemins sont le sien » (p. 147). Ces caractéristiques que l'auteur développe dans son essai La Vía del tarot sont en accord avec les vers qu'il compose sur cette Arcane initiale.

63. Alejandro Jodorowsky, «El Loco », Yo el tarot, dans Poesía sin fin, op. cit., p. 221

64. Ibid., v. 1-4: «J'avance dans le chant abominable des formes/messager de l'essentiel, de même moi/dédaignant les rêves de la pensée/je fais de tous les chemins, mon chemin.» (traduction personnelle).

65. Nous reprenons avec ce terme ce qu'Ana Maria Binet expose dans son travail sur Fernando Pessoa dans lequel elle montre comment l'imagination active ou créatrice « met en branle tout le processus de transfiguration du réel ». Voir Ana Maria Binet, L'Ésotérisme dans l'œuvre de Fernando Pessoa, op. cit., p. 349-350.

66. Alejandro Jodorowsky, Viaje esencial, Madrid, Siruela, 2016.

67. Alejandro Jodorowsky, La Vía del tarot, op. cit., p. 39.

68. Carl Gustav Jung, Commentaire sur le Mystère de la Fleur d'Or, Paris, Albin Michel, 1979.

69. Alejandro Jodorowsky, "Libertad interior », Viaje esencial, op. cit., p. 181, v. 1-8: "Prisonnier de la loi commune, sois libre de tes entrailles./Interdiction et ordres fleurissent aux superficies, dans/ton crâne réside l'insondable./La conscience est une barque qui navigue sur la mer de la folie/Parce que tu es mortel, tu es libre./Que dans ton corps enchaîné danse le squelette !/ Allaité de morales piérreuses, sauras-tu te dissoudre/dans l'infini? » (traduction personnelle).

70. Mathias Auclair, «A la recherche d'un art total », La revue de la BNF, numéro 37, 2011/1, p. 23-30.

71. Cette dualité, que nous n'avons pas exploitée pleinement si ce n'est par l'évocation de l'ambivalence entre la lumière et l'obscurité de l'abîme, est l'un des principaux points communs des œuvres à caractère ésotérique. L'alchimie notamment s'accompagne toujours de couples dans sa symbolique : le roi et la reine, masculin et féminin, souffre et mercure. Carl Gustav Jung en prendra exemple et définira l'anima et l'animus dans la Dialectique du moi et de l'Inconscient en 1933 (publié chez Gallimard, Paris).

72. En effet, la définition même de l'ésotérisme se base sur l'existence de correspondances entre les religions, les textes et les cultures. Pour Antoine Faivre, c'est l'un des quatre éléments fondamentaux qui permet de définir une œuvre comme appartenant à l'ésotérisme: «Il existerait des correspondances symboliques et réelles (point de place, ici, pour l'abstraction) entre toutes les parties de l'univers visible et invisible ("ce qui est en haut est comme ce qui est en bas, et comme ce qui est en haut..."). On retrouve de là l'antique idée du microcosme et du macrocosme ou, si l'on préfère, le principe d'interdépendance universelle. » Antoine Faivre, L'Ésotérisme, Paris, PUF, « Que-sais-je », 1992, p. 14.

\section{RÉSUMÉS}

Le caractère pluridisciplinaire de l'œuvre d'Alejandro Jodorowsky est l'un des éléments fondamentaux de son parcours d'artiste. Les productions cinématographiques, les bandes dessinées ou encore ses apports sur le tarot sont autant de particularités qui ont fait sa popularité. Sa poésie surgit dans les années 2000 avec la publication de ses premiers recueils, pourtant, Jodorowsky adoptait déjà un comportement poétique dans les années 1960 lors de la création de Panique ou en 1973 lorsqu'il portait à l'écran La Montagne sacrée (La Montaña sagrada). Dans cet article nous montrerons comment la poésie est au cœur de son art et fait le lien entre 
ses nombreuses productions. La poésie pour Alejandro Jodorowsky est en effet avant tout une pensée et un acte qui, conjointement, permettent la naissance de ses multiples pièces. L'acte poétique apparaît déjà à l'artiste au Chili dans les années 1950, cette philosophie de la poésie se consolidera ensuite dans les années 1960 et 1970 en France.

Alejandro Jodorowsky's multidisciplinary aspect is one of the fundamental elements of his artistic journey. The cinematographic productions, the comics or yet again his contributions on Tarot are as many particularities that make him known to many. The poetry arose in the 2000's with the publication of his first collections, yet, but it was already present in the 60's during the creation of "Panic" or in 1973, when Jodorowsky brought The Holy Mountain to the screens. In this article, we will demonstrate how poetry is the heart of his art and the unifying link of his many productions. Poetry, for Alejandro Jodorowsky, is indeed and above all a reflection and an act, that conjointly, allowed the birth of his numerous plays. The poetic act already appears to the artist in Chile during the 50's, this philosophy of poetry will then consolidate itself during the 60's and 70's in France.

\section{AUTEUR}

\section{JOY COURRET}

Doctorante en Études hispaniques à l'Université de Bordeaux Montaigne, Joy Courret prépare une thèse sur l'ésotérisme et la spiritualité dans l'œuvre poétique d'Alejandro Jodorowsky. Elle a participé à plusieurs colloques au sujet de la poésie d'Alejandro Jodorowsky, notamment à Montpellier (23 mars 2018), à Lisbonne (30 octobre 2018) ou encore à Bruxelles lors du festival « Humains en sociétés » en décembre 2017. 